\title{
Viel öfter Behandlungsfehler als gedacht
}

\author{
Bessert sich das Asthma bei Kindern nicht, muss es nicht unbedingt daran liegen, dass die Medikation \\ nicht mehr ausreicht. In den allermeisten Fällen liegen die Fehler woanders, wie eine Studie ergab.
}

Bevor bei Kindern mit unkontrolliertem Asthma die Dosis der Dauermedikation erhöht wird, sollte man prüfen, ob das grundlegende Asthma-Management wirklich leitliniengerecht erfolgt: Daran erinnern Ärzte um Dr. Eric P. de Groot vom PrinzessinAmalia-Kinderzentrum im niederländischen Zwolle.

Sie haben die Krankenakten von Kindern analysiert, die wegen eines vermeintlich therapierefraktären Asthmas an eine Spezialklinik überwiesen worden waren. Nur in knapp drei Prozent der Fälle war eine Therapieresistenz bestätigt worden (Acta Paediatrica, online: 30 Juni 2015).

Alle 142 Kinder und Jugendlichen im Alter zwischen fünf und 17 Jahren hatten vor dem Klinikkontakt ein inhalatives Kortikosteroid (ICS) angewendet, jeder Fünfte in Kombination mit einem lang wirksamen Betamimetikum.

Bei 138 (97,2\%) von ihnen förderten Anamnese und Untersuchung behebbare Ursachen für die fehlende Asthmakontrolle zutage. Der häufigste Grund war bei 53 Kindern (37,3\%) mangelnde Therapieadhärenz.

40 Kinder $(28,2 \%)$ waren nach ihrer Diagnose weiterhin in relevantem Maße Triggerfaktoren ihres Asthmas ausgesetzt, meist in Form von Aeroallergenen und Zigarettenrauch.

Die unzureichende Behandlung von Begleiterkrankungen hatte bei 28 Kindern (19,7\%) den Behandlungserfolg behindert; bei den Komorbiditäten handelte es sich um allergische Rhinitiden und dysfunktionelle Atmung.

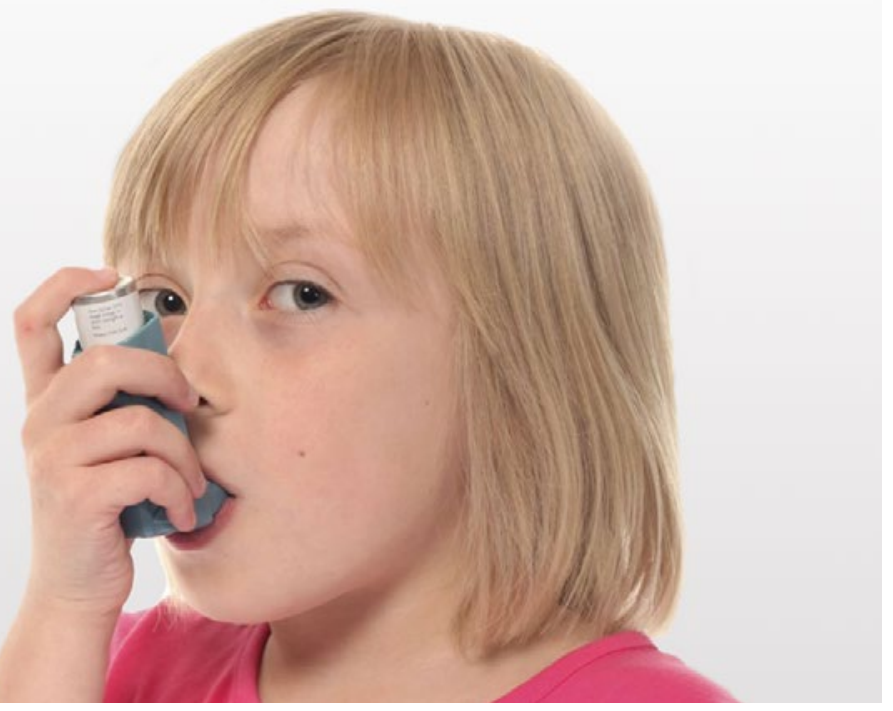

...in einer weiteren Untersuchung wendeten Kinder Inhalatoren oft falsch an.

\section{Falscher Umgang mit Inhalatoren}

Elf Patienten (7,8\%) beherrschten nicht einmal das Handwerkszeug der Therapie, sie wendeten die Inhalatoren nicht richtig an. Und bei weiteren sechs Kindern (4,2\%) war die Asthmatherapie deswegen erfolglos geblieben, weil sie gar kein Asthma hatten.

Vielmehr bestand bei vier von ihnen eine dysfunktionelle Atmung, eines litt an einer allergischen Rhinitis und ein weiteres an einem chronischen Husten in Zusammenhang mit einer massiven häuslichen Tabakrauchexposition.

Durch eine Korrektur der Behandlung konnte bei allen 138 Kindern eine - zumindest teilweise - Kontrolle des Asthmas erreicht werden. Die Tagesdosis an ICS wurde dabei insgesamt sogar etwas reduziert, dafür erhielten mehr Patienten als zuvor eine Kombinationstherapie.

In den zwei Jahren nach der Therapieanpassung kam es bei 16 Prozent der Patienten zu einer Asthmaexazerbation, sechs Prozent mussten stationär behandelt werden.

Ähnliche Mängel im grundlegenden Asthmamanagement wurden laut de Groot und Kollegen auch in anderen Studien aufgedeckt. So konnten etwa bei Kindern, die an das britische Zentrum für problematisches Asthma überwiesen wurden, durch Hausbesuche in 80 Prozent der Fälle derartige Fehler identifiziert werden.

Einer anderen Untersuchung zufolge bleibt eine allergische Rhinitis bei Asthmakindern häufig unerkannt und unbehandelt. Bei dieser Komorbidität könnte nach Einschätzung von de Groot ein intranasales Kortison helfen, die Asthmakontrolle zu verbessern.

Beate Schumacher

\section{PCl-Patienten: Fast jeder vierte hat schweres Schlafapnoe-Syndrom}

KHK-Patienten, die sich einer Angioplastie unterziehen müssen, leiden überraschend häufig an einem schweren obstruktiven Schlafapnoe-Syndrom (OSA). Die OSA fällt bei diesen Patienten zuvor vergleichsweise selten durch exzessive Tagesschläfrigkeit auf und wird im sog. „Berlin Questionaire“ häufig nicht erfasst. Das sonst bewährte Diagnose-Tool eignet sich folglich nicht für Herzpatienten, resümiierten die Studienautoren beim ATS-Kongress. Sie hatten 1305 KHK-Patienten, die in Brasilien, China, Indien, Singapur oder Myanmar im Herzkatheterlabor behandelt worden waren, in einem Schlafstudio untersuchen lassen. Man fand eine obstruktive Schlafapnoe (OSA) bei nicht weniger als $45 \%$ der Patienten. Bei 21,8\% wurde eine schwere OSA (AHI >30) diagnostiziert. Nur ein Viertel der Patienten berichtete über erhebliche Tagesschläfrigkeit, nur 54\% der Patienten wurden durch den Berlin-Fragebogen aufgedeckt.

Quelle: Kongress der American Thoracic Society (ATS) vom 15-20. Mai in Denver 